Cinémas

Revue d'études cinématographiques

Journal of Film Studies

\title{
Un fracassant Big Bang
}

\section{Edmond Couchot}

Volume 1, numéro 3, printemps 1991

Nouvelles technologies : nouveaux cinémas?

URI : https://id.erudit.org/iderudit/1001063ar

DOI : https://doi.org/10.7202/1001063ar

Aller au sommaire du numéro

Éditeur(s)

Cinémas

ISSN

1181-6945 (imprimé)

1705-6500 (numérique)

Découvrir la revue

Citer cet article

Couchot, E. (1991). Un fracassant Big Bang. Cinémas, 1(3), 7-20.

https://doi.org/10.7202/1001063ar

\section{Résumé de l'article}

L'auteur démontre ici que la continuité entre photographie, cinéma, télévision et images de synthèse n'est en fait qu'apparente. Elle se fonde sur le progrès technique alors que l'image de synthèse, davantage qu'un progrès technique, inscrit une rupture radicale dans le système de figuration en cours depuis cinq siècles, instaurant un nouvel ordre visuel qui ne représente plus le réel mais le simule. L'image numérique substitue au monde réel un monde purement virtuel. Devant la crise que l'image numérique fait subir au cinéma, à la télévision, à la photographie, l'auteur voit un déplacement de ces arts en vue d'une hybridation avec le numérique, une voie d'avenir qui renouvelle l'imaginaire. 


\title{
Un fracassant Big Bang
}

\section{Edmond Couchot}

\begin{abstract}
RÉSUMÉ
L'auteur démontre ici que la continuité entre photographie, cinéma, télévision et images de synthèse n'est en fait qu'apparente. Elle se fonde sur le progrès technique alors que l'image de synthèse, davantage qu'un progrès technique, inscrit une rupture radicale dans le système de figuration en cours depuis cinq siècles, instaurant un nouvel ordre visuel qui ne représente plus le réel mais le simule. L'image numérique substitue au monde réel un monde purement virtuel. Devant la crise que l'image numérique fait subir au cinéma, à la télévision, à la photographie, l'auteur voit un déplacement de ces arts en vue d'une hybridation avec le numérique, une voie d'avenir qui renouvelle l'imaginaire.
\end{abstract}

\begin{abstract}
The author regards the similarities between photography, cinema, television and image synthesis as more apparent than real. The perceived continuity is based on technical progress. But image synthesis is more than simply another instance of technical improvement. It marks a radical break with the system of figuration used for five centuries and heralds the coming of a new visual order in which the real world is not so much represented as simulated. Digital imaging substitutes a virtual world for the real world. The crisis in cinema, television, and photography resulting from the advent of digital imaging will lead, the author argues, to a shift towards the digital, towards a renewal of imagining.
\end{abstract}

Le terme de «nouvelles technologies» appliqué au domaine de la création visuelle entretient une fâcheuse confusion. On trouve pêle-mêle, sous cette appellation, les derniers avatars techniques de la photo, du cinéma et de la télévision, ainsi que, bien sûr, les 
images de synthèse. Certains même considèrent la photographie comme la première des nouvelles technologies et postulent ainsi une sorte de continuité dans le progrès technique qui évoluerait jusqu'aux techniques numériques, le cinéma prolongeant la photographie, la télévision prolongeant le cinéma, l'image de synthèse prolongeant la télévision. En réalité, l'écart technologique qui sépare une image télévisée d'un tableau de la Renaissance, construit selon les règles de la perspective à projection centrale, est beaucoup plus petit que celui qui sépare une image télévisée d'une image de synthèse donnant à voir un objet ou une scène apparemment identiques. L'image de synthèse est bien plus qu'un progrès technique, elle introduit dans le système de figuration en cours depuis cinq siècles une rupture radicale, sans précédent historique. Avec elle, un nouvel ordre visuel apparaît qui bouleverse de fond en comble notre économie symbolique.

Projeter sur l'image de synthèse les grilles d'analyse de la photo, du cinéma ou même de la télévision reste inefficace pour saisir la spécificité technique et, dans une certaine mesure, la spécificité esthétique vers lesquelles elle tendrait ${ }^{1}$. Aussi bien, la question du retentissement possible des nouvelles technologies de l'image sur le cinéma n'a de pertinence qu'à deux conditions: considérer que seules les technologies numériques (ou de la synthèse d'image) sont vraiment «nouvelles», c'est-à-dire en rupture avec les techniques traditionnelles; et mettre en évidence les caractéristiques de cette rupture en réexaminant l'image cinématographique (et éventuellement l'image télévisée) à partir de l'image numérique.

\section{La Représentation optique et son automatisation}

Avec le cinéma, un des vieux rêves de l'humanité s'est réalisé: reproduire par l'image le mouvement des choses. La technique cinématographique s'inscrit dans une longue quête, celle de l'automatisation de l'image, qui commence (ou recommence) avec la "perspective à projection centrale» à la Renaissance, et se poursuit avec la photographie au XIXe siècle. Le cinéma ajoute à la photo la possibilité d'automatiser grâce au même appareil ${ }^{2}$ à la fois la synthèse et l'analyse du mouvement. Alors que la photographie n'avait su capturer qu'une trace figée du monde animé — et c'était déjà fabuleux —, le cinéma put redonner à voir indéfiniment le mouvement des choses dont la pellicule avait, une fois pour toutes, saisi l'image. L'automatisation du processus porte la logique visuelle d'un mode de figuration fondé sur une certaine organisation de la lumière - l'optique - à son efficacité maximale, mis à part le cinéma holographique qui ajoutera la perception de la troisième dimension. Ce mode de figuration 
profondément lié au devenir technologique de l'Occident soustend toute une économie symbolique, extraordinairement riche et variée dans sa complexité et ses contradictions, qu'on peut désigner globalement comme celle de la Représentation optique.

Le principe en est simple: l'image est morphogénétiquement générée par la projection lumineuse sur un plan bidimensionnel d'une réalité qui lui préexiste. Dans la perspective telle qu'elle a été pratiquée à partir du Quattrocento, l'image était formée par projection optico-géométrique sur un plan d'intersection coupant la pyramide visuelle constituée à sa base par les contours de l'objet et à son apex par l'œil du peintre. Cette projection introduisait dans la morphogenèse de l'image un automatisme très complexe qui libérait - pas encore totalement, mais d'une manière importante - la main et l'œil du peintre de tout un lourd travail, notamment de la construction de la profondeur et des échelonnements des figures qu'il n'avait su jusqu'alors correctement maîtriser. L'opération exigeait que le peintre eût en face de lui un objet, un visage, une scène réelle, que cette réalité et lui-même fussent exactement confrontés, distingués et unis par la même lumière, partageant le même espace et le même temps au moment de l'acte de figuration, même si la peinture voulait qu'il prît avec cette réalité une certaine distance et qu'il la recomposât à sa guise. Quand il arrivait au peintre de ne rien avoir sous les yeux et de n'emprunter qu'à sa vision intérieure, la perspective lui permettait alors de projeter ces objets imaginaires sur le plan du tableau comme s'ils avaient eu la consistance et la présence du réel.

Avec la photographie, le procédé s'automatise encore davantage puisque l'image s'enregistre et se fixe dorénavant sans le recours de la main. Mais le principe technique essentiel de la figuration reste le même. Il existe un isomorphisme complet entre le système de projection optico-géométrique de la perspective (des dispositifs divers des chambres noires ou claires jusqu'aux règles géométriques) et l'appareil photographique le plus moderne. Dans tous les cas, la morphogenèse de l'image implique nécessairement la présence d'un objet réel préexistant à l'image. Elle crée une relation biunivoque entre le réel et son image. L'image se donne alors comme la représentation du réel. Elle en présente une empreinte lumineuse permanente, morphogénétiquement stable, capable de perdurer dans le temps et d'être présentée à nouveau - re-présentée - indéfiniment.

\section{Concomitance}

Mais l'image établit aussi une jonction entre deux instants du temps, celui où elle a été saisie et celui où elle est regardée. S'il 
s'agit, par exemple, du portrait peint d'un personnage, cet instant privilégié - constitué en réalité d'une somme d'instants - est celui où ce personnage est venu poser en personne devant le peintre $^{3}$. S'il s'agit d'une photographie, cet instant, lui aussi privilégié, sera celui de la pose où le photographe et l'objet auront été coprésents dans l'acte de figuration. La logique visuelle de la représentation permet ainsi non seulement de passer d'un point quelconque d'un espace à trois dimensions à son correspondant (son «transformé») dans un espace à deux dimensions, mais également d'un point de temps à un autre point de temps. Elle établit une relation particulière entre l'espace et le temps; elle les rend homogènes l'un à l'autre. Ce qui a pour conséquence de faire coïncider mentalement dans le temps et dans l'espace celui qui regarde l'image et celui qui l'a faite. Toute l'économie figurative de la culture occidentale se construira, à partir du Quattrocento, autour de ce système de communication particulier qui tend à faire coïncider le sujet imageur - celui qui enregistre ou peint l'image - et le sujet regardeur - dans la même relation d'affrontement épistémique à l'objet représenté, au réel: celui de l'alignement sur le même axe du sujet, de l'image et de l'objet ${ }^{4}$.

Le cinéma s'inscrit exactement dans la même logique et la renforce en automatisant l'analyse et la synthèse du mouvement ou plutôt de sa représentation. Il joue encore davantage sur la mise en concomitance de l'imageur et du regardeur puisque la prise de vue du mouvement implique une prise de temps, non plus instantanée mais étendue, séquentielle - le plan-séquence - donnant l'impression d'une continuité temporelle entre chaque photogramme. Mais dans le cinéma, comme le remarque Gilles Deleuze (p. 7-22), les instants séparant chaque photogramme ne sont plus des instants privilégiés, ils sont déterminés par un automatisme qui se limite à contrôler rigoureusement mais aveuglément les intervalles de temps séparant chaque arrêt de l'image. À l'instant décisif de la pose correspond la durée non moins décisive du plan. Il ne s'agit plus de capturer une infime fraction de la durée, comme dans la photo, mais de prélever dans son épaisseur même, d'en relever complètement l'empreinte en devenir. Il s'agit de plonger le regardeur dans l'écoulement d'un temps originaire (celui de la prise de vue) et de faire coïncider l'empreinte de la durée avec la durée propre, le temps subjectif du regardeur. Car de même qu'une photo ou qu'un tableau ne peuvent être vus qu'en position frontale (la tête de mort anamorphosée des Ambassadeurs d'Holbein le confirme), la pellicule cinématographique ne peut être visionnée que dans l'ordre im- 
muable où les photogrammes se succèdent puisque, une fois l'empreinte prise, celle-ci devient immuable. Cette concomitance spatiale et temporelle tend à immobiliser le regardeur et à le soustraire à tout événement extérieur susceptible d'introduire quelque perturbation temporelle dans le déroulement chronique du film. D'où la logique du dispositif cinématographique: la salle obscure qui rabat les regards sur l'écran vers lequel ils convergent en s'ignorant, la synchronisation à raison de vingtquatre images par seconde de l'horloge perceptive interne du spectateur avec celle du projecteur.

\section{Une adhérence au réel}

Tandis que la photographie attache le regardeur au réel au moyen d'un faisceau de lumière, qu'elle le rabat obstinément sur cet objet nécessairement préexistant, qu'elle le place sur l'axe défini par le réel et l'image - qu'elle le «fait adhérer» au réel, dira Barthes - , le cinéma le rabat sur la durée, au moyen d'un faisceau de temps, non pas d'une façon abstraite et générale mais singulière, unique, sur son écoulement événementiel, jamais reproductible intégralement (dix prises de la même scène ne sont jamais identiques), sur son irréversibilité absolue 5 , le cinéma fait adhérer le regardeur au temps.

La télévision, encore une fois, mais pour une dernière fois, va porter cette logique figurative à son point culminant. L'image générée sur fond de caméra électronique n'est pas différente morphogénétiquement de celle qui se projette sur une pellicule photographique ou sur un film. Elle reste toujours une "émanation» lumineuse d'une réalité préexistante saisie et organisée par l'objectif de la chambre noire. Ce qui change, en revanche, c'est le mode d'enregistrement de cette image. Contrairement à la photographie et au cinéma, où le plan entier de l'image projetée sur la plaque ou sur le film est enregistré d'un seul coup et d'une façon permanente (après sa fixation), la télévision opère d'abord par une analyse linéaire. L'image initiale (optique) est décomposée, par balayage, en une trame de fines lignes parallèles dont l'intensité lumineuse en chaque point est traduite par une modulation électronique - le signal vidéo. Pour restituer l'image, il faut en faire la synthèse, c'est-à-dire retraduire à son tour la modulation électronique en intensité lumineuse, grâce à un second balayage, propre au dispositif de réception, rigoureusement synchronisé avec le balayage du dispositif d'émission.

Or, cette synthèse a lieu, dans le cas du direct, au moment même où l'image est prise. Plus aucun délai entre le moment de la prise de vue - propre à l'image cinématographique - et le 
moment de la représentation ${ }^{6}$. Il y a désormais coïncidence temporelle, quasi-immédiateté, entre le moment de l'enregistrement et celui de la représentation. C'est dans ce court-circuit entre deux lieux, celui de l'émission et celui de la réception, et entre deux temps, celui de l'analyse et celui de la synthèse de l'image, que tient l'essentiel de la spécificité technique de la télévision. Allumer son poste de télévision, c'est d'abord se connecter sur le lieu de l'émission de l'image, au moment où elle se constitue, c'est être présent à la naissance même de l'image. Ce que ni la photo ni le cinéma ne permettent.

En ce sens, on ne peut pas dire que l'image de la télévision fonctionne exactement sur le mode de la simple re-présentation; elle opère sur un mode légèrement différent, mais qui s'inscrit dans la même logique: le mode de la surprésentation. C'est le dernier degré de la représentation. La surprésentation télévisuelle fait coïncider instantanément le temps de la réalité filmée dans son écoulement, celui de son image et celui du regardeur. Elle tend ainsi à provoquer une adhérence totale du sujet au temps événementiel. La télévision n'est plus une coupe mobile dans la durée, coupe rapportée ensuite par le transfert de la représentation, ce n'est plus un prélèvement détaché du temps, c'est l'incrustation de deux durées concomitantes mais qui ont lieu en deux lieux différents, le lieu où se tient le regardeur et le lieu où se tient l'objet. Alors que le spectateur cinématographique tend à s'évader de lui-même par effet de projection et d'identification, le spectateur télévisuel tend à se laisser saisir par un effet d'invasion, un mouvement du dehors vers le dedans, vers luimême. D'où la fascination du «direct» où l'on assiste de loin aux événements les plus spectaculaires et à leur irréversibilité fatale, en qualité de témoin impuissant jouissant de son impuissance? Que ce soit sur le mode de la représentation simple ou de la surprésentation, la logique de la Représentation optique tend à faire adhérer de plus en plus le regardeur au réel, à le référer de plus en plus étroitement à des fragments - topiques et chroniques préexistants de ce réel.

\section{Contraintes et détournement}

$\mathrm{Si}$ ce mode de figuration tient sa puissance des techniques elles-mêmes, l'esthétique (voire plus généralement l'économie symbolique, l'art et la culture) que ces techniques sous-tendent n'obéit pas à un strict déterminisme. Comme les astres, les techniques inclinent mais ne déterminent pas. Et c'est bien ce que nous montre l'histoire de l'art qui a toujours été un combat subtil entre les contraintes techniques avec lesquelles il fallait composer et leur détournement. Â commencer par la perspec- 
tive dont chaque peintre a réinventé une application singulière jusqu'à ce que le cubisme s'attaque directement au problème posé par la hiérarchie des plans introduit par le système perspectif. Tout se passe, en effet, avec le cubisme comme si la réalité, tenue jusqu'alors à distance de son image par les lois de la géométrie optique, venait s'écraser sur le tableau, se substituer à sa propre représentation (il en est ainsi, à la lettre, avec le collage), comme si elle se présentait elle-même. Et c'est bien ce qui caractérisera une très large part de l'art moderne: refuser toute re-présentation au profit d'une présentation directe qui renvoie la peinture à sa propre matière, faire coïncider le plus précisément possible le moment de la contemplation de l'œuvre avec le moment de l'acte de figuration, faire adhérer le regardeur à la réalité matérielle de la peinture et à la réalité événementielle de l'acte de figuration picturale et, dans la foulée, l'intéresser beaucoup plus au processus qu'au produit.

La photographie, de son côté, ne s'est pas contentée de reproduire le réel ni de s'en tenir au fameux moment décisif, elle a cherché à désaligner le regard du photographe de celui du regardeur. Elle a introduit de la mobilité dans la coupe instantanée de la pose, réinterrogé la notion de trace et d'enregistrement, voulu faire que ce «ça-a-été» n'ait pas été ou ait été autrement, que l'image actinique coupât toutes les amarres avec une quelconque réalité préexistante. Ce souci aura été aussi celui du cinéma qui a essayé, avec le montage, de rendre décisif non plus l'instant, comme la photo, mais la somme d'instants - une certaine durée - que constitue le plan, d'échapper à l'irréversibilité de l'enregistrement, de dilater ou de condenser le temps, de le représenter en reconstruisant les chaînes d'événements, leurs cascades, leurs ramifications. Souci encore de l'art vidéo, qui cherche à agir sur le temps même du balayage par désynchronisation, démodulation, à traiter l'image comme un fluide, ou inversement comme un objet dur et inerte, mobilier ou monumental, à neutraliser l'effet de surprésentation, à décaler, à mêler les sources, à confronter par incrustation des événements directs et enregistrés, le "ça-a-été» et le «c'est», à nier toute référence à la communication. Toutes les images fondées sur la logique de la Représentation optique caractéristique de la photo et du cinéma ou de la Surprésentation propre à la télévision témoignent de cette adhérence de l'image au réel, à une réalité préexistant dans l'espace et le temps, tout autant que d'une volonté obsessionnelle d'échapper à son attraction ${ }^{8}$. 


\section{La simulation numérique et le virtuel}

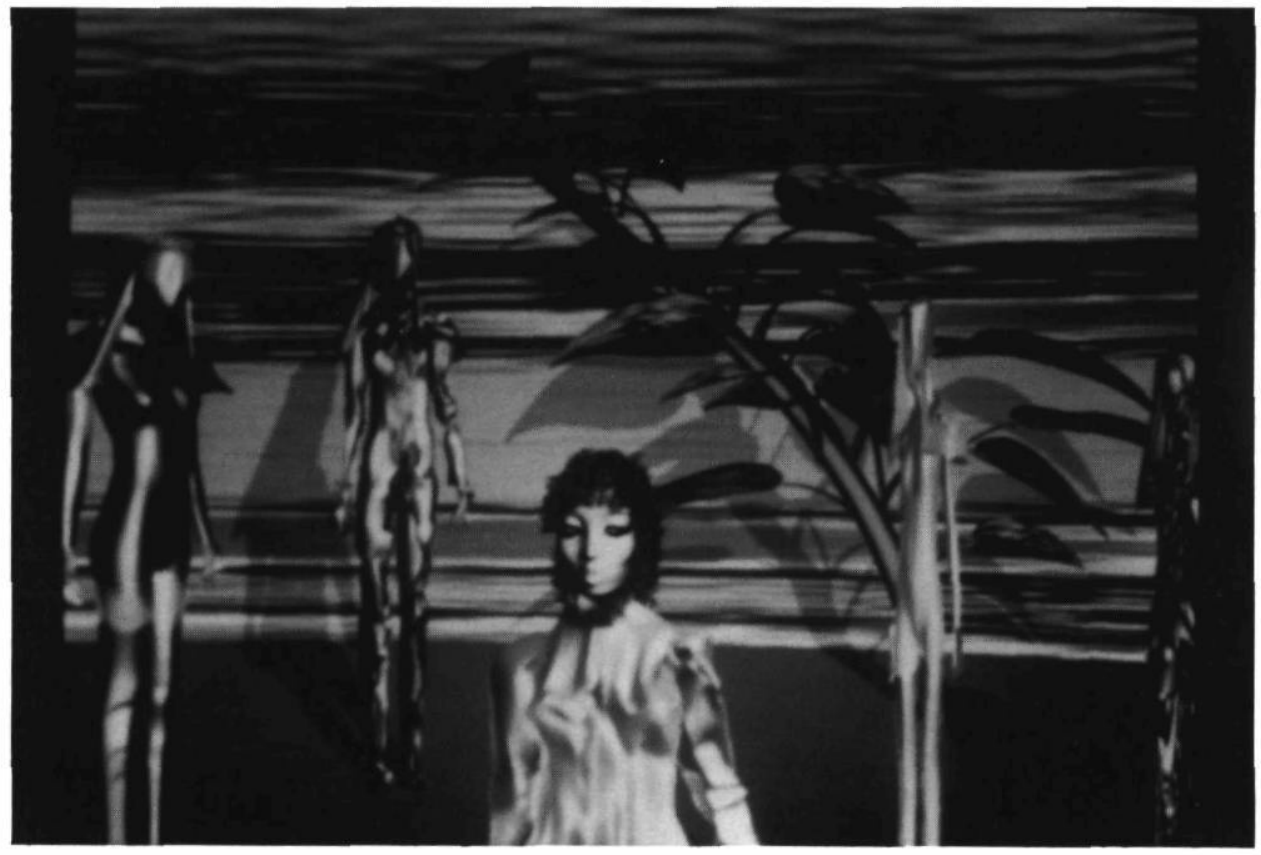

Pygmalion d'H. Huitric, M. Nahas, M. Saintourens, M.-H. Tramus

LUAP-Image, Université de Paris VII

Tout change, et radicalement, avec l'image numérique. Le rapport de l'image au réel, à l'espace et au temps, obéit à une autre logique. L'automatisation des processus d'analyse et de synthèse de l'image ne sont plus ceux du cinéma ni de la télévision, même si l'image numérique a encore besoin de leurs supports. Ces processus ne sont plus physiques - matériels ou énergétiques -, ils sont calculés. Ce qu'on peut comparer à l'analyse, d'abord, consiste à transformer en valeurs numériques les points d'images obtenues par des techniques traditionnelles (photo, cinéma, télévision, ou encore peinture, gravure, imprimerie, etc.). On procède, dans ce cas-là, à partir non pas d'une réalité brute dont on enregistre l'empreinte mais déjà d'une image complètement constituée 9 . Ainsi fonctionnent les palettes électroniques et diverses paint boxes. L'image qui s'affiche alors sur l'écran n'a plus aucun rapport direct avec quelque objet réel, quelque réalité préexistante que ce soit. Ce qui préexiste à cette image recomposée, synthétisée numériquement et projetée sur l'écran vidéo connecté à l'ordinateur, ce sont des nombres et 
seulement des nombres exprimés sous forme binaire dans la mémoire et les circuits du calculateur. L'image originaire est transformée en une matrice mathématique - elle est devenue une image-matrice - dont chacun des éléments constitue un «pixel». Réduits de la sorte à de simples nombres, les pixels seront très aisément manipulables. Il suffira pour agir sur eux de demander à l'ordinateur de changer leur valeurs chromatiques ou leurs positions sur l'écran (leurs coordonnées).

La spécificité de l'image numérique réside surtout dans la capacité de recréer l'intégralité de cet objet dans l'espace - et éventuellement l'intégralité de son mouvement - et non plus de reproduire l'apparence visuelle d'un objet fixe ou en mouvement à partir de son empreinte lumineuse, bref, elle réside dans la capacité de synthétiser le réel lui-même ou des fragments du réel. Ainsi, pour figurer le vol d'un oiseau, il faudra créer d'abord l'oiseau lui-même, le «modéliser", c'est-à-dire en décrire à l'ordinateur un modèle plus ou moins complet, plus ou moins proche de la réalité ${ }^{10}$, que celui-ci donnera à voir sous un angle, un éclairage ou à une échelle définis, puis à décrire avec la même exactitude ses mouvements dans l'air et la trajectoire de son vol. Alors que le fusil de Marey visait un oiseau réel pour en saisir l'empreinte, l'ordinateur recrée le réel, il le synthétise, non pas exactement ex nihilo, mais à partir d'une simulation du réel. L'image numérique n'est donc plus l'enregistrement sur un support chimique ou magnétique de la trace lumineuse laissée par un objet, elle est le résultat d'un processus où le calcul se substitue à la lumière, le traitement de l'information à celui de la matière et de l'énergie. À la logique de la Représentation optique succède la logique de la Simulation numérique.

Cette recréation du réel n'est évidemment qu'approchée, mais la logique de la simulation tend à l'accomplir avec le maximum de précision, selon des lois rationnelles qui le décrivent ou l'expliquent. Elle cherche à recréer de toutes pièces une réalité autonome, avec toute sa profondeur structurelle et fonctionnelle. Pour faire voler l'oiseau, par exemple, on pourra se contenter de décrire les mouvements de ses ailes, instant par instant, en s'aidant d'une étude cinématographique (oiseau préexistant), mais on pourra aussi synthétiser ce vol à partir d'un modèle inspiré d'observations scientifiques rationalisées et formalisées qui rendra l'opération autonome. On pourra encore aller plus loin et faire voler des oiseaux en groupe - ou déplacer des poissons en bancs - en laissant le soin à l'ordinateur de calculer chaque trajectoire et de faire en sorte que les oiseaux ou les poissons s'évitent tout en se dirigeant vers un but commun. 


\section{Un temps-matrice}

Si l'image-matrice est effectivement le résultat d'un calcul, ce calcul est lui-même une opération résultant du déroulement d'un programme mettant en œuvre des modèles de simulation extrêmement variés qui ne sont pas tous des modèles issus de la logique formelle ${ }^{11}$. Or, ces modèles sont déjà des interprétations de la réalité passées au crible de la rationalité scientifique; le réel qu'ils se proposent de reconstruire est un réel sans substrat matériel - hors le brouillard électronique des milliards de microimpulsions qui courent dans les circuits électroniques de l'ordinateur -, un réel qui n'a désormais d'existence que virtuelle. En ce sens, l'image de synthèse n'a dès lors plus aucune adhérence au réel: elle s'en libère. Elle n'est plus comme la photo, le cinéma, la télévision, ni même la peinture, projetée sur un écran ou un tableau; elle est éjectée du réel, avec assez de force pour l'arracher à son attraction et au champ de la Représentation. Elle ne renvoie plus à un réel originaire, brut et infiniment complexe, mais à un réel purifié au feu du calcul, débarrassé de ses scories, littéralement exorcisé, arraché à son destin premier.

Cet univers simulé change profondément les propriétés de l'espace dans lequel se tient la réalité virtuelle et les rapports de l'artiste à celle-ci. L'espace virtuel n'est ni l'espace physique où baignent nos corps et circule notre regard, ni l'espace imaginaire créé par le cerveau. C'est un espace sans lieu déterminé, où toutes les dimensions, toutes les topologies, toutes les lois d'associations, de déplacements, de translations, de projections, sont possibles: un espace utopique. Et conjointement, le temps virtuel associé à cet espace est un temps qui se tient hors de toute chronie, de tout présent, passé ou futur: un temps uchronique. Ce temps de synthèse n'est pas non plus celui du monde physique, celui du monde vivant, ou celui de notre pensée et de nos perceptions. Il en est exactement ainsi lorsque les modèles de simulation sont utilisés dans certains dispositifs interactifs permettant une sorte de dialogue immédiat - dit en «temps réel» — entre l'image (et le programme qui la génère) et le regardeur, comme les simulateurs de vol bien connus où l'image est créée au cours même de son visionnement par les manipulations du pilote ${ }^{12}$. C'est un temps-matrice ouvert, sans orientation particulière, sans fin ni début (comme le film photographique ou la modulation scandée de la vidéo), qui s'autogénère, se réinitialise au gré du regardeur, selon les lois imaginées par l'auteur. Le temps uchronique n'est plus un temps habité par des événements, mais par de pures éventualités. Au «ça-a-été» de la représentation ou 
au «c'est» de la surprésentation se substitue le "ça-peut-être» de la simulation. L'image-matrice nous libère d'une certaine fatalité, quelles que soient les causes qui la font naître, déterministes ou chaotiques. Et cette libération ne passe plus essentiellement par la conscience pure (l'intuition du libre arbitre), l'imaginaire et l'art, mais par la technologie.

Voilà, à mes yeux, le problème de fond que pose la simulation numérique: celui de l'automatisation technologique de la liberté. C'est sans doute l'un des plus importants que la pensée des hommes - n'ayons pas peur de paraître emphatique! - ait eu à résoudre. Il concerne, plus que tous les autres, les artistes, puisque ceux-ci situent leur action au croisement de l'imaginaire et du réel, de la liberté et de la contingence. Que feront-ils de cette nouvelle et étrange liberté? Il faut remarquer d'abord que si la simulation numérique est capable de créer un monde où tout est «virtu-réellement» possible, tout n'y est cependant pas prêt à prendre automatiquement sens. Et c'est tant mieux! Car le mode de figuration fondé sur la simulation, si radicalement différent qu'il soit de celui fondé sur la représentation (ou sur sa négation), reste cependant dans une large mesure indissociablement lié à ce dernier.

En effet, le paradoxe de la simulation est de pouvoir simuler la représentation, le paradoxe du numérique de simuler l'analogique. C'est pourquoi tant d'images numériques semblent ne pas se distinguer des images traditionnelles; elles mettent d'ailleurs souvent une certaine coquetterie à entretenir cette confusion et à se faire passer pour ce qu'elles ne sont pas, ce qui leur est la plupart du temps préjudiciable. Le passage de l'ordre visuel de la représentation optique à celui de la simulation numérique ne peut avoir de signification qu'à la condition d'une certaine continuité qui perdure dans ce changement brutal et qu'à celle que notre culture à venir ne soit pas, elle aussi, éjectée de la culture présente et passée. Aussi bien, devrait-on se diriger plutôt vers des solutions hybrides que vers des solutions «tout simulation». Il serait vain d'annoncer la mort du cinéma, de la télévision, de la photographie, ou de la peinture, bien qu'il soit difficile de nier la crise qui les frappe, mais il faut bien constater que ces moyens de figuration - tous destinés, à court ou à moyen terme, à être numérisés - sont technologiquement dépassés par le numérique et destinés à perdre leur hégémonie. Leur seul avenir est soit dans le déplacement, soit dans l'hybridation. Le déplacement consisterait à réaffirmer autrement leurs spécificités techniques et leurs esthétiques: une sorte de retour aux sources remontant au-delà des sources. Ce que fit la peinture en inventant l'impressionnisme pour répondre à la photographie; ce 
que fit le cubisme et une partie de l'art moderne en portant la logique de la représentation à son extrême ou en la traitant par la négation.

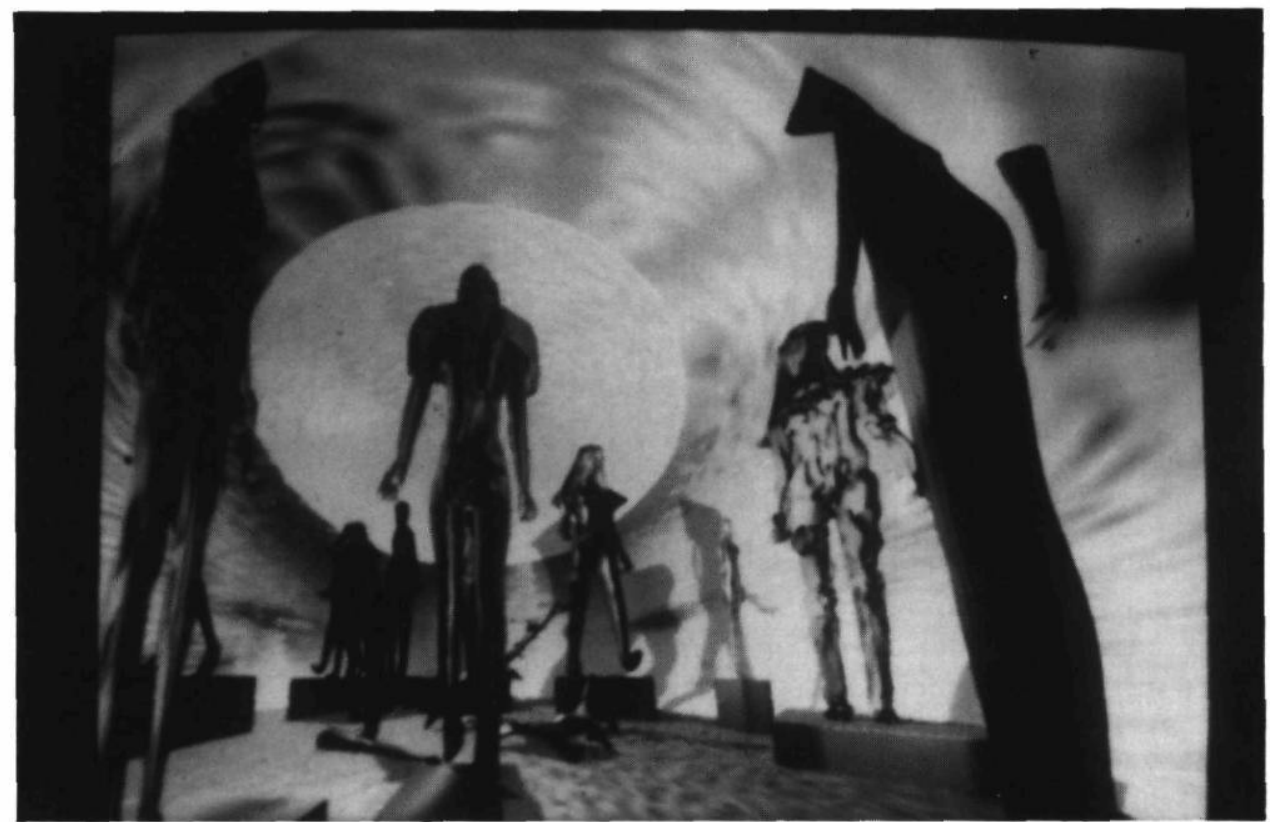

Pygmalion d'H. Huitric, M. Nahas, M. Saintourens, M.-H. Tramus

LUAP-Image, Université de Paris VII

La voie de l'hybridation consiste à imaginer un art - des arts, car il ne s'agit pas seulement de l'image, susceptibles d'interfacer le virtuel et le réel. Hybridations internes, au sein de l'immense réservoir des modèles numériques qui génèrent les images de synthèse. Hybridations externes entre les techniques traditionnelles et les techniques de synthèse, hybridations entre toutes les catégories d'images, hybridations entre les différentes logiques figuratives, tant au plan de la morphogénèse (la fabrication de l'image et de ses formes) qu'au plan de la distribution (la diffusion, la transmission, la reproduction, la conservation, la manipulation, bref, la socialisation de l'image). La numérisation de la télévision à l'échelle planétaire inclinera vraisemblablement vers de telles hybridations. Déjà, les techniques numériques ont permis à certains artistes venus des arts plastiques, comme Hervé Huitric et Monique Nahas, Michel Bret, David Em, Yoichiro Kawaguchi et d'autres, d'explorer les nouvelles possibilités de 
l'image de synthèse sans refaire de la peinture, du cinéma ou de la vidéo. D'autres viennent de la vidéo, comme Lillian Schwartz, Steina et Woody Vasulka, et plus récemment Zbigniew Rybzynski qui propose, avec Steps et L'Orchestre, des images qu'il était impossible d'obtenir avec des procédés classiques et qui expriment magistralement ce que pourra devenir un art de l'hybridation. Du côté du cinéma, les exemples sont plus rares mais la numérisation du montage cinématographique laisse entrevoir un renouvellement important du septième art et de sa distribution, telle est du moins l'opinion du cinéaste Jean-Pierre Beauviala ${ }^{13}$. Tout un art des réseaux et de la connexion (Fred Forest, Brian Ruffin-Smith, Roy Ascott), des dispositifs interactifs où non seulement le réel et le virtuel mais aussi l'image et le regardeur s'interfacent et s'hybrident (Jeffrey Shaw - The legible city -, M. W. Krueger - Video place -, P. Broadwell -A nano sample -, V. J. Vincent - Mandala -, J.-L. Boissier - Le Bus -, E. Couchot avec la collaboration de M. Bret et M.-H. Tramus - La Plume et le pissenlit -, etc.) commence à émerger.

Mais si la voie de l'hybridation passe obligatoirement, comme nous l'avons dit, par cette paradoxale possibilité propre à l'image de synthèse de simuler n'importe quelle catégorie d'images (avec plus ou moins d'approximation, certes), elle passe aussi par un renouvellement de l'imaginaire. L'ouverture d'une liberté virtuelle sur l'infini n'est que théorique, du moins je le souhaite, car rien ne serait pire pour un artiste que de jouir, comme certains le disent, de la puissance illimitée du démiurge. Les artistes de la simulation ne sont pas des démiurges; ou si l'on veut garder le terme grec, il faut l'employer dans son sens premier, celui d'artisan (qui travaille pour le peuple) et non de dieu. Tout au plus, travaillant sur des signes, seraient-ils des "sémiurges». À cette liberté sinon illimitée, du moins fantastiquement agrandie par rapport à la liberté que tout artiste connaissait face à la manipulation du réel, il faut opposer maintenant de nouvelles contraintes puisqu'il n'est pas d'art sans contraintes. Des contraintes qui soient capables de dessiner des limites, des bornes, donc une forme, non pas à l'imagination mais à ce nouvel univers virtuel de l'utopie illimitée et de l'uchronie absolue dont on assiste au fracassant Big Bang. 


\section{NOTES}

1 Bien qu'il soit à la mode de considérer que les images n'ont plus guère de spécificités et que cette perte de résistance entre des images générées par des techniques différentes - véritable perte de «résistance immunitaire» permettant tous les passages, les mélanges et les collages possibles, n'appelle pas d'explication profonde.

2 Du moins à l'origine puisque l'appareil inventé par les frères Lumière, le «cinématographe», permettait, sans modification, d'enregistrer et de projeter l'image. Ces appareils se sont ensuite diversifiés mais ils fonctionnaient selon le même principe technologique depuis près d'un siècle.

3 Il faut remarquer que l'art du portrait s'est développé en même temps que la perspective qui semblait s'appliquer plutôt aux paysages ou aux constructions architecturales.

4 Voir à ce propos mon article «Sujet, Objet, Image», «Nouvelles images, nouveau réel», Cahiers internationaux de sociologie, vol. LXXXII (janvierjuin 1987).

5 On ne peut qu'inverser les sens du film. Le ralentissement ou l'accélération n'apportent que des transformations analogiques à la durée originaire.

6 Sauf, évidemment, s'il s'agit d'un enregistrement, où nous retrouvons le cas du cinéma.

7 Les réalisateurs tentent de compenser cette impuissance avec les émissions interactives qui font appel à la participation du public au moyen du téléphone.

8 C'est lorsque cette volonté de non-représentation est la plus forte, comme dans l'art abstrait, qui veut ne renvoyer directement à aucune réalité préexistante explicite, que la logique de la représentation atteint son efficacité maximale.

9 Certaines «saisies» d'objets réels peuvent se faire par l'intermédiaire de dispositifs tridimensionnels capables de reconstituer leur volume.

10 On peut aussi utiliser des modèles complètement imaginaires qui ne font aucune référence au réel.

11 La part des mathématiques, toute relative, doit donc être reconsidérée.

12 Le temps uchronique peut, en revanche - de même que l'espace utopique de la simulation est susceptible de simuler l'espace réel et ses lois - , simuler n'importe quel type de temps, le temps dit réversible de l'astrophysique, le temps entropique orienté vers le désordre ou le temps néguentropique du vivant orienté vers l'ordre et la complexité.

13 Voir en particulier «Entretien avec Jean-Pierre Beauviala», Sonovision 307 (septembre 1987).

\section{OUVRAGE CITÉ}

Deleuze, Gilles. Cinéma 1. L'image-mouvement. Paris: Minuit, 1983. 